\title{
Quark Confinement and Surface Critical Phenomena *
}

\author{
K.J. Juge ${ }^{\mathrm{a}}$, J. Kuti ${ }^{\mathrm{b}}$ and C.J. Morningstar ${ }^{\mathrm{b}}$ \\ ${ }^{a}$ Fermi National Accelerator Laboratory, P.O. Box 500, Batavia, Illinois 60510 \\ ${ }^{\mathrm{b}}$ Dept. of Physics, University of California at San Diego, La Jolla, California 92093-0319
}

Surface critical phenomena and the related onset of Goldstone modes probe the fundamental properties of the confining flux in Quantum Chromodynamics. New ideas on surface roughening and their implications for lattice studies of quark confinement are presented. Problems with the oversimplified string description of the Wilson flux sheet are discussed.

\section{The spectrum of the confining flux}

A rather comprehensive determination of the rich energy spectrum of the confined chromoelectric flux between static sources in the fundamental representation of $\mathrm{SU}(3)_{c}$ was reported earlier [1,2] for separations $\mathrm{r}$ ranging from $0.1 \mathrm{fm}$ to $4 \mathrm{fm}$. The full spectrum is summarized in Fig. 11 with different characteristic behavior on two scales separated approximately at $\mathrm{r}=2 \mathrm{fm}$.

\section{No string spectrum for $\mathrm{r} \leq \mathbf{2} \mathrm{fm}$}

The $\Sigma_{\mathrm{g}}^{+}$ground state is the familiar static quark-antiquark potential which is dominated by the rather dramatic linearly-rising behavior once $\mathrm{r}$ exceeds about $0.5 \mathrm{fm}$. Although the empirical function $\mathrm{E}_{\Sigma_{\mathrm{g}}^{+}}(\mathrm{r})=-\mathrm{c} / \mathrm{r}+\sigma \mathrm{r}$ approximates the ground state energy very well for $r \geq 0.1 \mathrm{fm}$, the fitted constant $\mathrm{c}=0.3$ has no relation to the running Coulomb law whose loop expansion breaks

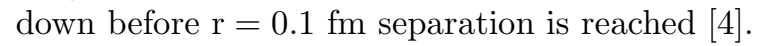
Early indoctrination on the popular string interpretation of the confined flux for $\mathrm{r} \leq 2 \mathrm{fm}$ was mostly based on the observed shape of the $\Sigma_{\mathrm{g}}^{+}$ground state energy: the linear shape of the ground state potential for $\mathrm{r} \geq 0.5 \mathrm{fm}$ and the approximate agreement of the curvature shape for $\mathrm{r} \leq 0.5 \mathrm{fm}$ with the ground state Casimir energy $-\pi /(12 \mathrm{r})$ of a long confined flux [5]. The excitation spectrum clearly contradicts earlier claims [3] on the simple string interpretation of the linearly

\footnotetext{
*Presented by J. Kuti at Lattice 99, Pisa, June 29 - July 3, 1999. Supported by DOE Grant DE-FG03-97ER40546. UCSD/PTH/99-19, FERMILAB-Conf-99/279-T
}

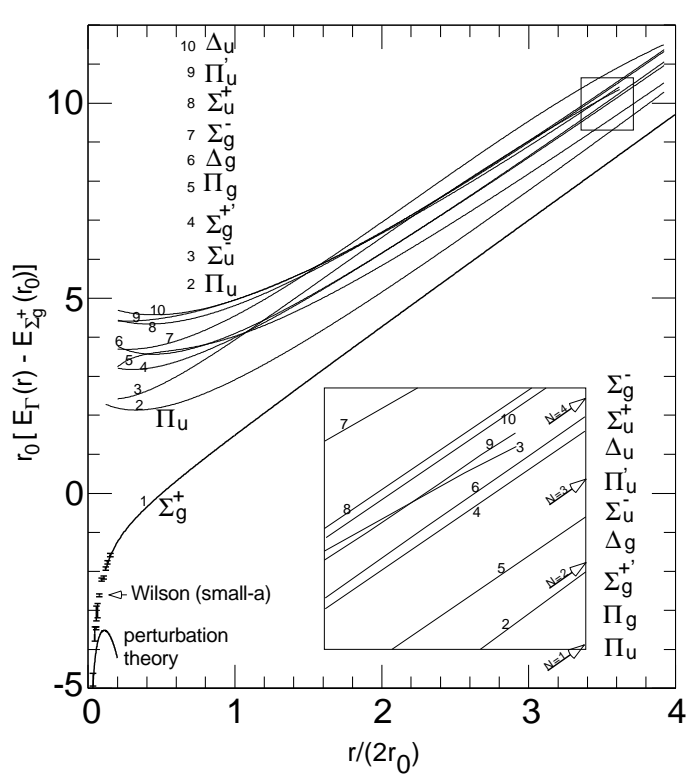

Figure 1. Continuum limit extrapolations are shown for $r_{0}\left[E_{\Gamma}(r)-E_{\Sigma_{g}^{+}}\left(r_{0}\right)\right]$ against $r /\left(2 r_{0}\right)$ for ten different states characterized by quantum numbers $\Gamma$. The arrows in the inset show the locations of the four lowest string modes and the associated discrepancies in the spectrum (for notations and further discussions, see Refs. [1],2]).

rising confining potential. The gluon excitation energies lie well below the string predictions and the level orderings and degeneracies are in violent disagreement with expectations from a fluctuating string. 


\section{Goldstone modes for $r>2 \mathrm{fm}$}

A feature of any low-energy description of a fluctuating flux sheet in euclidean space is the presence of Goldstone excitations associated with the spontaneously-broken transverse translational symmetry. These transverse modes have energy separations above the ground state given by multiples of $\pi / r$ (for fixed ends). The level orderings and approximate degeneracies of the gluon energies at large r match, without exception, those expected of the Goldstone modes. However, the precise $\pi / \mathrm{r}$ gap behaviour is not observed. The spectrum is consistent with massive capillary waves on the surface of the flux sheet, with a cutoff dependent mass gap. The most likely explanation for this gap is Peierls-Nabarro lattice pinning of the confining flux sheet at small correlation lengths. Our new results [6] on the same spectrum in $\mathrm{SU}(2)$ for $\mathrm{D}=3,4$, and a detailed test of the strong coupling spectrum in $\mathrm{SU}(2)$ for $\mathrm{D}=3$ lend further support to the above summary of the earlier findings.

\section{2. $\mathrm{Z}(2)$ model at $\mathrm{D}=3$}

The purpose of the $\mathrm{Z}(2)$ project is to understand flux formation and the string excitation spectrum from high statistics simulations and the loop expansion on the analytic side. The WKB approximation of flux formation in the sine-Gordon field representation of the monopole plasma was discussed earlier [7]. The Z(2) model maps into the Ising model by duality transformation which was exploited before in the study of large Wilson loops [8]. By invoking universality, the critical region of the $\mathrm{Z}(2)$ model is mapped into $\mathrm{D}=3 \Phi^{4}$ scalar field theory in the study of flux formation. The confining flux sheet of the Wilson loop corresponds to a twisted surface in the Ising representation which is described by a classical soliton solution of the $\Phi^{4}$ field equations. Excitations of the flux are given by the spectrum of the fluctuation operator $\mathcal{M}=$ $-\nabla^{2}+\mathrm{U}^{\prime \prime}\left(\Phi_{\text {soliton }}\right)$ where $\mathrm{U}(\Phi)$ is the field potential energy of the $\Phi^{4}$ field. The spectrum of the fluctuation operator $\mathcal{M}$ of the finite surface is determined from a two-dimensional Schrödinger equation with a potential of finite extent [7]. In

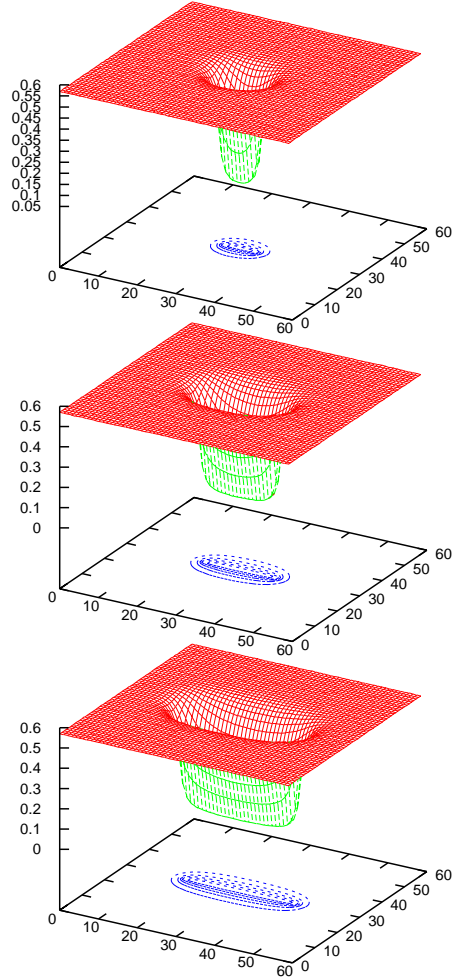

Figure 2. Flux formation at corr. length $\xi \approx 2$ for spatial Wilson loop sizes $r=10,20,30$.

the limit of asymptotically large surfaces, the equation becomes separable in the longitudinal and transverse coordinates. The transverse part of the spectrum is in close analogy with the quantization of the one-dimensional classical $\Phi^{4}$ soliton. There is always a discrete zero mode in the spectrum which is enforced by translational invariance in the transverse direction. Figs. 2, 3, 1,5 illustrate some of the results which are consistent with our findings in $Q C D$.

\section{Acknowledgements}

One of us (J. K.) would like to acknowledge valuable discussions with S. Renn, P. Hasenfratz, and F. Niedermayer on surface Goldstone modes. 


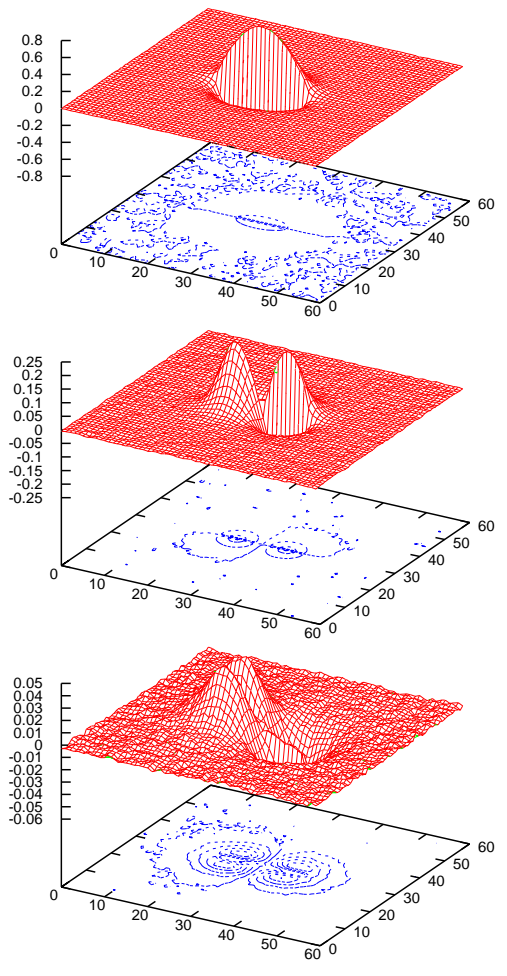

Figure 3. Wavefunctions of the excited $\mathrm{Z}(2)$ flux at spatial Wilson loop size $\mathrm{r}=20$ for $\xi \approx 2$. The first two Goldstone modes and a massive intrinsic excitation are shown.

\section{REFERENCES}

1. K.J. Juge, J. Kuti, and C. Morningstar, Nucl. Phys. B (Proc. Suppl.) 63, 326 (1998).

2. K.J. Juge, J. Kuti, and C. Morningstar, Nucl. Phys. B (Proc. Suppl.) 73, 590 (1999).

3. S. Perantonis and C. Michael, Nucl. Phys. B 347, 854 (1990)

4. M. Peter, Nucl. Phys. B 501, 471 (1997).

5. M. Lüscher, Nucl. Phys., B180 (1981) 317.

6. K.J. Juge, J. Kuti, and C. Morningstar, to be published.

7. J. Kuti Nucl. Phys. B (Proc. Suppl.) 73, 72 (1999) and to be published.

8. M. Caselle, R. Fiore, F. Gliozzi, Nucl. Phys., B486 (1997) 245.

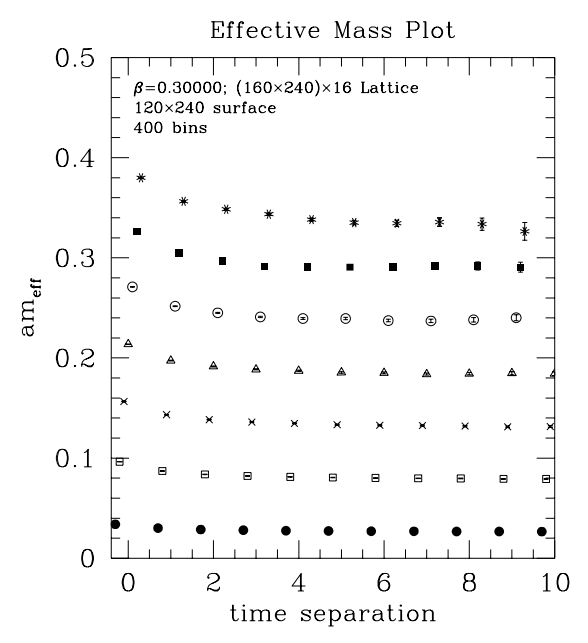

Figure 4. Effective mass plot of the string spectrum for a very large Wilson loop at $\xi \approx 1 / 2$ before the Peierls-Nabarro gap opens up.

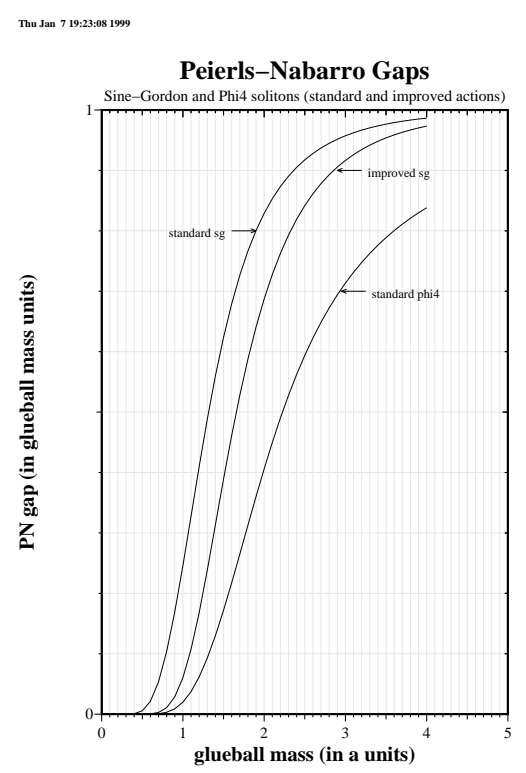

Figure 5. The Peierls-Nabarro gap (negligible at large correlation lengths, or inverse glueball mass) implies pinning and mass generation for the Goldstone modes. 